\title{
SEGURIDAD ALIMENTARIA EN COLOMBIA \\ Y MODELO RASCH
}

\section{FOOD SECURITY IN COLOMBIA AND RASCH MODEL}

\author{
Martha Inés Camargo G. (1), Doris Cristina Quintero L. (2), Oscar Fernando Herrán F. (2,3)
}

(1) Secretaria de Salud. Gobernación de Cundinamarca, Colombia.

(2) Observatorio Epidemiológico de Enfermedades Cardiovasculares. Centro de Investigaciones Epidemiológicas,

Universidad Industrial de Santander, Bucaramanga, Colombia.

(3) Escuela de Nutrición y Dietética. Universidad Industrial de Santander, Colombia.

\begin{abstract}
This study subjected two scales used to determine food security in national nutritional status surveys in Colombia (ENSIN) to the Rasch model to assess their performance on the contradictory evidence of its usefulness. In practical terms, the EPSA measures food insecurity based on a single item, p1, which due to its low difficulty (8.46 logits, standard deviation of 1.83) is inappropriate to measure the surveyed households. Based on statistical calculation five of the twelve items presented over fitting and three of the twelve (including the first) presented measurement degradation. Like the EPSA, p1 defines the result to a large extent showing that the test is inadequate for the surveyed households. Based on statistics at least four of the fifteen items showed over-adjustment and four of the fifteen, including the first, showed measurement degradation. Measurements using tools that are not appropriate, but attractive, can be a new and serious problem that adds to other conditions associated with household food insecurity.
\end{abstract}

Key words: Food security; reproducibility of results; validity of the test; hunger; Colombia.

Este trabajo fue recibido el 14 de Noviembre de 2011 y aceptado para ser publicado el 20 de Abril de 2012.

\section{INTRODUCCIÓN}

El avance tecnológico y el desarrollo de métodos estadísticos han propiciado el desarrollo de pruebas simplificadas para abordar la medición de temas complejos en las ciencias sociales, la educación y la salud $(1,2)$. La seguridad alimentaria (SA) y su complemento la inseguridad alimentaria (INSA) a nivel del hogar son conceptos complejos, aceptados como variables latentes, que tradicionalmente son estimados con métodos rigurosos pero poco viables debido a la logística y costos necesarios (3-5). Han sido varios los esfuerzos por obtener métodos de medición que aproximen estas condiciones a nivel del hogar, en algunos la simpleza en su aplicación y los bajos costos derivados los hacen atractivos (1-5). Sin embargo, como en cualquier prueba el desarrollo debe incluir análisis de reproducibilidad, validez y confiabilidad de sus resultados (1).

El análisis Rasch es una alternativa aceptada para evaluar el desempeño de pruebas en donde la medición se relaciona estrechamente con variables latentes relacionadas con las ciencias de la educación y sociales $(6,7)$, más recientemente este tipo de análisis ha sido usado en otros campos como el de la salud y la nutrición, donde las variables latentes son cercanas al campo de la psicometría y el comportamiento humano (1). El Rasch permite superar la principal crítica a los análisis realizados con la teoría clásica de las pruebas; "no es posible establecer una medida aditiva y lineal para medir variables latentes", en este caso la seguridad alimentaria (6).

En Latinoamérica incluida Colombia, se ha venido promocionado el uso de escalas simplificadas para medir la SA, en 2005 la Encuesta Nacional de la Situación Nutricional (ENSIN) utilizó la escala de percepción de la seguridad alimentaria (EPSA), y en la ENSIN 2010 la escala Latinoamericana y Caribeña (ELCSA) $(8,9)$. Varios estudios han mostrado resultados contradictorios cuando se comparan los resultados de estas escalas contra los de métodos referentes $(10,11)$. Además, 
pobre reproducibilidad y validez que comprometen su utilidad como pruebas simplificadas (11). En Colombia existe inequidad, pobreza e INSA en magnitudes que las convierten en problemas sociales y de salud $(8,9,12)$. En la política pública prima la óptica de que la SA es una variable dependiente y resultante del desarrollo estructural y social $(4,12)$, por lo tanto la mala clasificación o la imprecisión al calificar a un individuo u hogar puede llevar a intervenciones equivocadas o inespecíficas, lo que conlleva despilfarro de recursos públicos y dilución del impacto esperado en intervenciones poblacionales (13). El estudio de tecnología diagnóstica como la EPSA y la ELCSA es necesario dada la sensibilidad social sobre la SA, la INSA y la política pública derivada o asociada (12).

El objetivo de este estudio fue evaluar el desempeño de la EPSA y la ELCSA como tecnología diagnóstica para establecer la SA en hogares colombianos de diferentes estratos socioeconómicos y zonas geográficas, utilizando el modelo Rasch.

\section{SUJETOS Y MÉTODO}

Este estudio se clasifica como de evaluación de tecnología diagnóstica (1) y fue desarrollado durante 2009-2011 en cuatro ciudades de Colombia, Suramérica. Se realizó en tres etapas a) selección de la muestra, b) aplicación de formatos, c) análisis Rasch.

Selección de la muestra. Para que el hogar fuera elegible debía residir en la zona con dos años o más de anterioridad. En el área rural, dos veredas fueron seleccionadas al azar en cada municipio -Tenjo y Sibaté-, todos los hogares elegibles de esas veredas que aceptaron participar se encuestaron. Los hogares urbanos de Bogotá y Bucaramanga incluida su área metropolitana fueron seleccionados por muestreo aleatorio estratificado en múltiples etapas. Bogotá es la capital del país, Bucaramanga una ciudad intermedia y Tenjo y Sibaté municipios rurales articulados a Bogotá como proveedores de alimentos. Las manzanas de la ciudad se clasificaron en seis estratos socioeconómicos de acuerdo con la metodología de la oficina de planeación municipal, aleatoriamente seis manzanas fueron seleccionadas en cada estrato y sus mapas actualizados, las viviendas en ellas fueron numeradas en orden consecutivo. En los hogares del área rural que aceptaron participar y en los elegidos al azar se indagó por el jefe del hogar o por la persona responsable de la compra de los alimentos.

Cuando se evalúan pruebas se acepta que una muestra adecuada puede ser considerada como 10 multiplicado por el número de reactivos de éstas, para la EPSA una muestra de 120 hogares y para la ELCSA de 150 hogares serían apropiadas (14). Con este tama- ño muestral la significancia de la carga factorial de un reactivo se logra entre 0,40 y 0,45 -alfa 0,05 , poder de 0,80 y error estándar dos veces mayor que el coeficiente de correlación tradicional.

Fuentes de información. Tres instrumentos fueron aplicados. Un formato para recoger información sociodemográfica del hogar y los individuos, y las escalas EPSA y ELCSA que permiten medir a nivel del hogar la SA en el último mes. El jefe del hogar respondió el formato para recoger datos sociodemográficos del hogar, luego respondió las dos escalas asignando la primera mediante aleatorización simple.

La EPSA es una escala compuesta por doce ítems, con respuestas dicotómicas para cada uno y complementos en una escala de 1 a 3 que permiten establecer la severidad de la INSA, en los hogares donde no hay menores de dieciocho años la escala se limita a siete ítems (8). La ELCSA es una escala de quince ítems, en los hogares donde no hay menores de dieciocho años la escala se limita a diez ítems, todos dicotómicos (9), si la respuesta a los primeros cuatro ítems es negativa la ELCSA considera al hogar seguro y termina la aplicación de la misma (9).

Análisis Rasch. El modelo Rasch permite obtener en una escala estandarizada diferentes estadísticos para aproximarse al desempeño de una prueba en función de la habilidad del que es medido (hogar) y de la dificultad del ítem con que se mide, el modelo se resume así; $\log _{\mathrm{e}}($ Pni/(1-Pni $)=\mathrm{B}_{\mathrm{n}}-\mathrm{D}_{\mathrm{i}}$; donde Loge es el logaritmo natural, (Pni/(1-Pni) es la razón de dos probabilidades "odds"- dificultad del ítem/habilidad del hogar -, y $\mathrm{B}_{\mathrm{n}}$ y $\mathrm{D}_{\mathrm{i}}$ son distancias en logits relativas al origen local de la variable latente, en este caso la seguridad alimentaria $(6,7)$. El logaritmo del odds es conocido como "logit". Para establecer si el ordenamiento o concatenación de ítems presentado en las pruebas se correspondía con su probabilidad de respuesta dada su dificultad, se calculó para cada ítem el logaritmo natural de la razón entre las probabilidades complementarias o logit, que en Rasch es la unidad de medida de la measure, la measure o habilidad también fue calculada para los hogares. El ordenamiento de los ítems es una aproximación a la validez del constructo $(6,7)$. La relación anterior fue graficada para establecer visualmente el balance entre la habilidad de los hogares y la pertinencia de las pruebas dada la dificultad de sus ítems (Hogar-Map-Ítem) $(6,7)$.

También fueron calculados estadísticos de ajuste para el modelo. Las estadísticas de ajuste son las medias cuadradas de los residuales ó diferencias estandarizadas y se denominan infit y outfit. El infit o ajuste con información ponderada o también próximo y el outtfit o ajuste sensible a los casos atípicos o también lejano, varían 
entre cero e infinito y dependiendo del tipo de prueba (diagnóstica o de tamizaje) existen diferentes criterios para aceptarlos, éstos van en rangos de 0,8 a 1,2 o de 0,5 a 1,5. Un valor se considera extremo si es superior a 2,0 y debe interpretarse como que el ítem mide con excesiva imprecisión, degradando el conjunto de las otras medidas, valores inferiores de 1 deben interpretarse como que los residuales observados son menores a los esperados y representa sobreajuste. El sobreajuste aporta poco a la medición pero no la degrada, produciendo artificialmente buena fiabilidad (reability), confiabilidad o reproducibilidad (reliability) y altos índices de separación $(6,7)$.

La fiabilidad en este contexto para los hogares debe entenderse como la confiabilidad alcanzada en el orden de la medida hecha en el hogar, en esta muestra y con este conjunto de ítems, en otras palabras, la capacidad de reproducir la clasificación del hogar. La fiabilidad se computa como $\left(\mathrm{SD}^{2}-\mathrm{RMSE}^{2}\right) / \mathrm{SD}^{2}$; donde $\mathrm{SD}^{2}$ es la desviación estándar de la measure para los hogares y el $\mathrm{RMSE}^{2}$ la raíz cuadrada del error del modelo. La fiabilidad o reproducibilidad para los ítems es la capacidad para obtener el mismo nivel de dificultad en el ítem con el conjunto de ítem y en la muestra estudiada $(6,7)$. En pruebas como las evaluadas es necesario un mínimo de fiabilidad de 0,8 para obtener clasificaciones confiables. La alta fiabilidad de los ítems no compensa la falta o baja de fiabilidad en la clasificación de los hogares. Complementariamente, el índice de separación es dependiente de la fiabilidad y es calculado como (SD verdadera/RMSE), si el error estándar decrece aumenta la separación $(6,7)$.

Otro aspecto evaluado fue la dimensionalidad de las escalas. Una dimensión en este contexto es una variable latente; la "seguridad alimentaria", el "hambre" o la "incapacidad de acceso físico a los alimentos". Las dimensiones o subdimensiones en una escala son obtenidas al descomponer la varianza explicada por el modelo Rasch usando análisis de componentes principales, un componente es un factor, y para no confundir la interpretación clásica de lo que es un componente, el término equivalente usado en Rasch es el de contraste. Si lo esperado es un amplio rango de habilidades en los hogares -todos los estratos y áreas urbanas y rurales- y un amplio rango de dificultad en los ítems de una prueba, una varianza explicada pequeña podría indicar que la prueba es mala $(6,7)$. Para evaluar la dimensionalidad además de calcular la varianza explicada por el modelo y descomponerla para diferentes contrastes, se graficó la dificultad de los ítems (measure) versus sus valores de carga factorial "eigen" (15), con el fin de examinar la pertinencia de la agrupación de las preguntas o ítems (p). Varianzas inexplicadas mayores de $2 \%$ en el primer contraste indican nuevas dimensiones $(6,7)$.

Calidad de los datos. Todas las encuestas, fueron realizadas por Nutricionistas Dietistas entrenados durante dos semanas en los métodos utilizados. Las encuestas fueron revisadas diariamente por los encuestadores y semanalmente por supervisores de campo. Todos los datos fueron traducidos a "bits" con un lector óptico [Teleform]. Todas las bases de datos fueron sometidas a procesos sucesivos de verificación y limpieza con algoritmos lógicos hasta asegurar que los datos se correspondían con los escritos. Dado el diseño de la muestra la estimación de los errores se realizó utilizando el ajuste de la varianza de Taylor (16). El procesamiento de datos junto con el análisis fue realizado con STATA 10,1 SE StataCorp, 2008 (17). El análisis Rasch fue realizado con WINSTEPS versión 3,72. (18). La descripción de las variables numéricas incluyó el intervalo de confianza del 95\% (IC 95\%).

El estudio fue aprobado por el Comité de Ética en Investigación de la Facultad de Salud de la Universidad Industrial de Santander. Todos los participantes dieron su consentimiento informado por escrito.

\section{RESULTADOS}

En total se visitaron 1185 hogares, se declararon elegibles $621(52,4 \%)$ y de éstos, respondieron las encuestas 432 hogares $(69,6 \%)$. En Bogotá 151 hogares, en Bucaramanga y su área metropolitana 99, en Tenjo 91 al igual que en Sibaté. La tasa de participación en el área urbana fue de 60,8\%, en la rural de $85,7 \%$. El intervalo entre aplicaciones de la EPSA fue de 14,8 días (IC 95\%; 13,1 a 16,4) y para la ELCSA de 13,5 días (IC $95 \% ; 12,6$ a 14,5). Debido a que los valores estimados no se modificaron al corregir por el efecto del diseño de la muestra $(\mathrm{deff}=4)$, los reportados son similares a los obtenidos de un muestreo aleatorio simple.

Características de los hogares estudiados. El $57,9 \%$ de los hogares pertenecen al área urbana, el $55,2 \%$ de los sujetos habitan en éstos, sin diferencia en el número de integrantes, que en promedio fue de 3,6 (IC 95\%; 3,4 a 3,8) en el área urbana y de 3,8 (IC 95\%; $3,5$ a 4,1$)$ en la rural, $p=0,183$. En promedio cada hogar urbano tiene un menor de 18 años (IC 95\%; 0,9 a 1,2), el rural 1,4 (IC 95\%; 1,2 a 1,6), p=0,004. En el 62,2\% de los hogares hay menores de edad, sin diferencias por el área geográfica o ciudad estudiada, p>0,05. El 9,2\% de los hogares en la zona urbana y el $12,1 \%$ en la rural tienen menos de un año de permanencia en el municipio, el 73,1\% en la urbana y el 78,6\% en la rural tres o más años en el municipio. Otras características de los hogares se presentan en la tabla 1.

Mapa de hogares e ítems (Ver apéndice 1). La 
figura 1 muestra sobre el eje $\mathrm{Y}$ en una escala lineal con base en el logaritmo de las probabilidades "logit", la relación que existe entre la percepción del jefe con respecto a la INSA en su hogar (habilidad del hogar para tener SA) y las preguntas utilizadas para aproximarse a la INSA (severidad o dificultad de los ítems). Los hogares con mayor puntuación se ubican en la parte superior del eje Y, mientras los de más baja en la parte inferior. Tanto para el mapa de hogares (izquierda del eje) y de ítems (derecha del eje) la letra $\mathrm{M}$ representa el promedio, la S la primera desviación estándar y la T la segunda des- viación estándar. La habilidad de los hogares según la EPSA estuvo entre - 4,85 y 4,68 logits, para la ELCSA entre - 6,91 y 5,99. La dificultad para los ítems según la EPSA estuvo entre - 8,46 y 2,74 logits, para la ELCSA entre $-5,83$ y 4,11. Ítems con valores de +- 2 logits son indeseables en las escalas por no aportar a la medición y producir sobreajuste artificial o degradación de la medida. La relación hogar-ítem predominante en las dos escalas es sesgada negativa, lo que traduce inhabilidad de los hogares para contar con SA dado los ítems utilizados para evaluarla; los ítems en las escalas presentan mayor

\section{TABLA 1}

Características de los hogares estudiados.

\begin{tabular}{|c|c|c|c|c|}
\hline Variable & $\begin{array}{l}\text { Total } \\
{[432]}\end{array}$ & $\begin{array}{l}\text { Urbano } \\
\text { [250] }\end{array}$ & $\begin{array}{l}\text { Rural } \\
{[182]}\end{array}$ & Valor $\mathbf{p}$ \\
\hline Edad del jefe & $\begin{array}{l}44,8 \\
(43,4 \text { a } 46,6)^{*}\end{array}$ & $\begin{array}{l}45,7 \\
(44,0 \text { a } 44,7)\end{array}$ & $\begin{array}{l}43,6 \\
(41,1 \text { a } 46,6)\end{array}$ & 0,147 \\
\hline Sexo del jefe & & & & 0,012 \\
\hline Hombre & $70\{16,2\} \dagger$ & $50\{20,0\}$ & $20\{11,0\}$ & \\
\hline Mujer & $362\{83,8\}$ & $200\{80,0\}$ & $162\{89,0\}$ & \\
\hline Escolaridad & & & & 0,000 \\
\hline Analfabeta/primaria & $152\{35,2\}$ & $50\{20,0\}$ & $102\{56,0\}$ & \\
\hline Hasta secundaria & $152\{35,2\}$ & $85\{34,0\}$ & $67\{36,8\}$ & \\
\hline Técnico/universidad & $115\{60,6\}$ & $115\{46,0\}$ & $113\{7,2\}$ & \\
\hline Nivel socioeconómico I & & & & 0,000 \\
\hline Uno & $276\{62,7\}$ & $94\{37,6\}$ & $177\{97,2\}$ & \\
\hline Dos & $106\{24,5\}$ & $103\{41,2\}$ & $3\{1,7\}$ & \\
\hline Tres & $55\{12,7\}$ & $53\{21,2\}$ & $2\{1,1\}$ & \\
\hline Tenencia de la vivienda & & & & 0,000 \\
\hline Propia sin deuda & $212\{49,1\}$ & $120\{48,0\}$ & $92\{50,6\}$ & \\
\hline Propia con deuda & $61\{14,1\}$ & $48\{19,2\}$ & $13\{7,1\}$ & \\
\hline Arriendo & $110\{25,5\}$ & $71\{28,4\}$ & $39\{21,4\}$ & \\
\hline Otra & $49\{11,3\}$ & $11\{4,4\}$ & $38\{20,9\}$ & \\
\hline Alcantarillado & & & & 0,000 \\
\hline $\mathrm{Si}$ & $306\{70,8\}$ & $250\{100,0\}$ & $56\{30,8\}$ & \\
\hline No & $126\{29,2\}$ & $0\{0,0\}$ & $126\{69,2\}$ & \\
\hline Inseguridad alimentaria & & & & 0,000 \\
\hline EPSA & $155\{35,9\}$ & $69\{27,6\}$ & $86\{47,2\}$ & \\
\hline ELCSA & $255\{59,0\}$ & $126\{50,4\}$ & $129\{70,9\}$ & \\
\hline
\end{tabular}


dificultad que la habilidad de los hogares. En las dos escalas el ítem 1 (p1) presenta excesiva menor dificultad de la esperada, que es entre - 2 y 2 logits. La EPSA en términos relativos a la ELCSA presenta menor dificultad entre un ítem y otro y menos ítems en desajuste (outfit $<0,5$ ó $>1,5), 5$ de 12 contra 10 de 15 respectivamente.

Dificultad de los ítems, validez de constructo y estadísticos de ajuste. Las tablas 2 y 3 presentan para cada escala el ordenamiento de los ítems de acuerdo con los valores de dificultad, y algunos estadísticos de ajuste interno (infit) y externo (outfit). El orden de los ítems de acuerdo con su dificultad difiere del orden presentado en las escalas -esperado-, este aspecto es substancial dado que en las escalas evaluadas se repiten saltos de un ítem difícil a uno menos difícil, lo que compromete la validez del constructo subyacente, es decir la SA y su complemento la INSA. El orden de los ítems observado en las dos escalas es consistente para el total y las dos áreas geográficas evaluadas.

Para la EPSA y con base en el outfit las respuestas en los ítems p1, p4, p10, p11 y p12 son predecibles originando sobreajuste. En el total de la muestra los ítems con sobreajuste son p1 y p9 (2 de 12), en el área urbana los ítems p1, p4 y p10 (3 de 12), en el área rural los ítems p1, p7, p9 y p11 (4 de 12). Complementariamente, en el total y las áreas urbana y rural los ítems p8 y p12 son improductivos para construir la medida-pobre ajuste- pero el valor extremo de p8 degrada el sistema de medición. Para la ELCSA y con base en el outfit las respuestas en los ítems p9, p5, p7, p8, p9, p10, p11,p13,

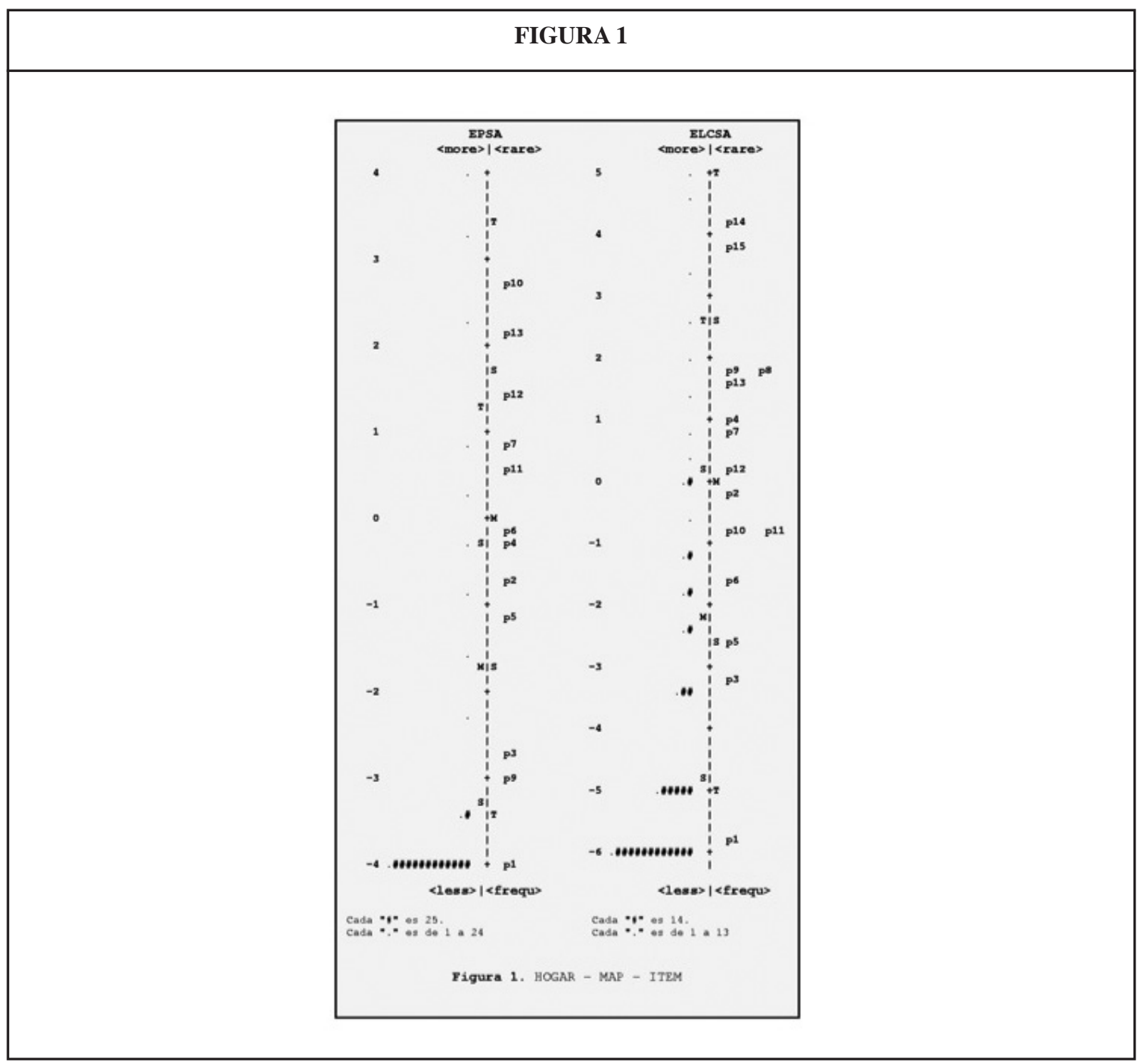




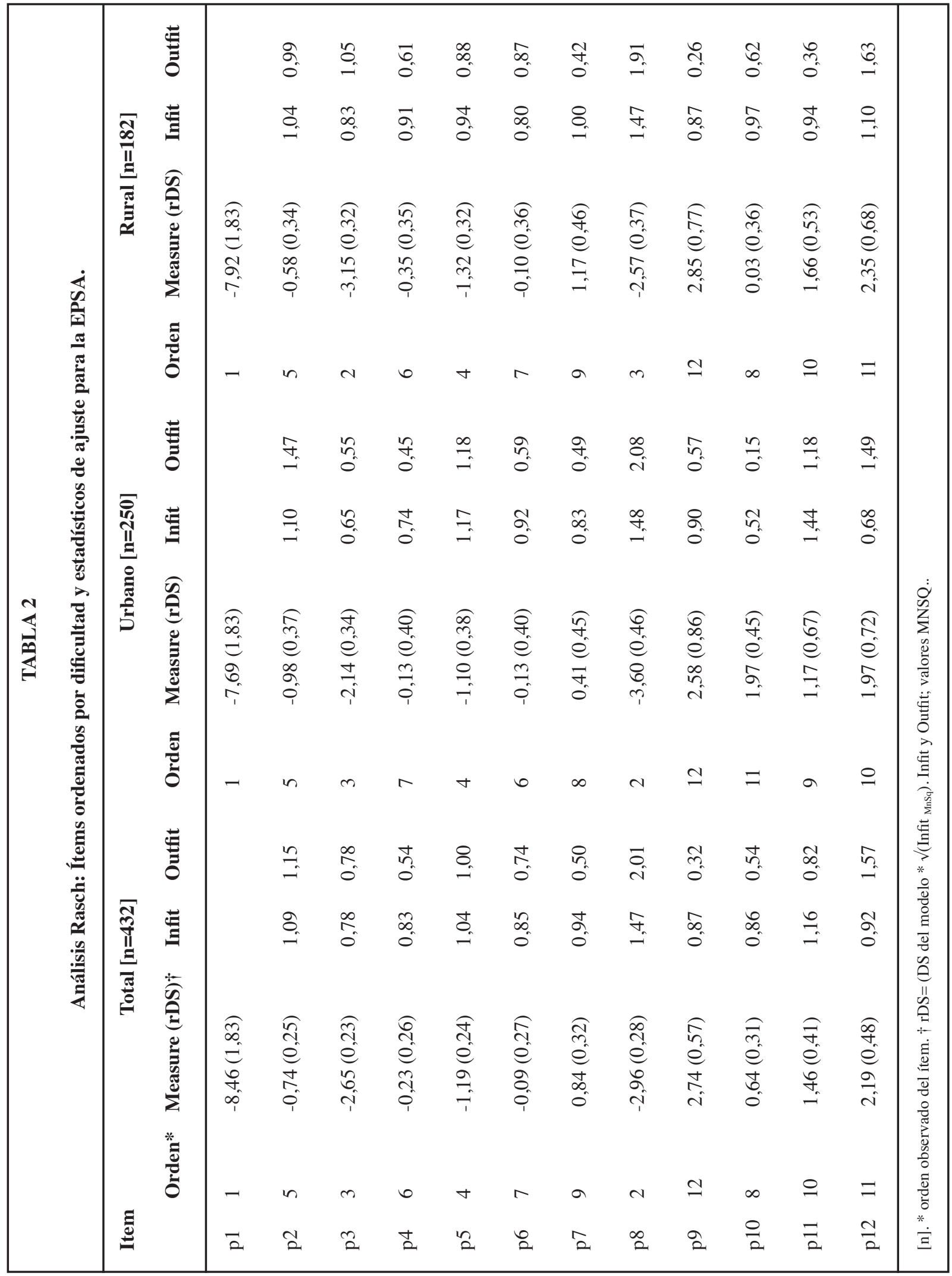




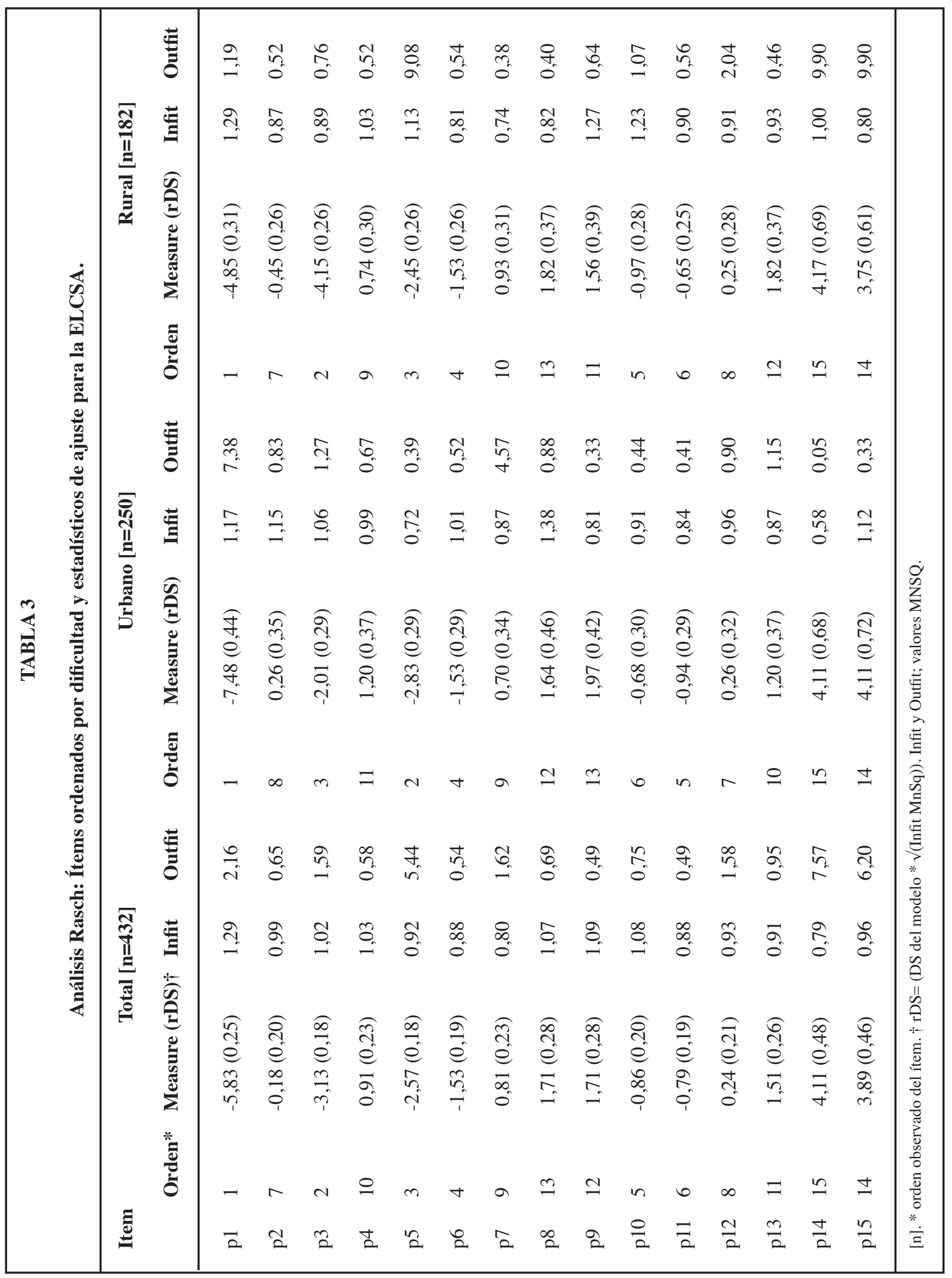


p14 y p15 son predecibles presentando sobreajuste. En el total de la muestra los ítems con sobreajuste son p9 y p11 (2 de 15), en el área urbana los ítems p5, p9, p10, p11, p14 y p15 (7 de 12), en el área rural los ítems p7, p8 y p13 (3 de 15). Complementariamente, en el total los valores extremos de p1, p5, p14 y p15 (4 de 15), y en las áreas urbana y rural los valores extremos en los ítems p1 y p7 (2 de 15) y p5,p12, p14 y p15 (4 de 15) son improductivos para construir la medida -pobre ajuste-, y degradan el sistema de medición. Todos los ítems tanto para la EPSA como la ELCSA están dentro del rango $>0,5$ y $<1,5$ según su valor infit (tablas 2 y 3 ).

La tabla 4 presenta el resumen de los estadísticos alcanzados tanto para los hogares como los ítems. En los hogares la habilidad media para tener SA es extremadamente baja en las dos pruebas, y la variabilidad de este estadístico es mayor a la deseable, en el área urbana es más evidente $-4,31$ para la EPSA y $-5,39$ para la ELCSA. Los valores medios de infit y outfit para los hogares están cerca del valor ideal de uno, sin embargo la variabilidad también es alta. El índice de fiabilidad para los hogares con la EPSA es de es 0,06 y de 0,74 con la ELCSA, consecuentemente el índice de separación es menor para la EPSA, 0,24 versus 1,67 de la ELCSA.
La dificultad media de los ítems para la EPSA es menor en términos relativos con los de la ELCSA en donde el valor medio se acerca al teórico ideal de cero, pero excediendo la variabilidad aceptable de +2 a -2 logits. Los valores outfit para los ítems en la ELCSA total y rural muestran valores que sobrepasan el límite teórico superior (mayor de 2,0), para las dos pruebas los ítems muestran alta fiabilidad superiores a 0,90 y valores extremos en el índice de separación para la EPSA de 4,50 y para la ELCSA de 9,32.

Dimensionalidad de la EPSA y ELCSA. La varianza explicada por la EPSA es de $44,7 \% ; 15,6 \%$ por los ítems y $29,1 \%$ por los hogares. La varianza explicada por la ELCSA de $61,1 \% ; 27,2 \%$ por los hogares y $33,9 \%$ por los ítems. Al descomponer la varianza se encontró que la inexplicada en el primer contraste para la EPSA fue de 2,1\% y para la ELCSA de 2,6\%. La figura 2 muestra el mapa de dimensionalidad para las dos escalas con base en sus cargas factoriales (valores eigen) y la dificultad de los ítems (measure). En la EPSA el mapa de dimensionalidad para el primer contraste no muestra nuevas dimensiones, en la ELCSA se configura una segunda con base en los ítems p14 y p15 (figura disponible solicitándola a los autores).

\section{TABLA 4}

Análisis Rasch: Resumen de las medidas de habilidad, dificultad, ajuste y confiabilidad.

\begin{tabular}{|c|c|c|c|c|c|c|}
\hline Estadístico & $\begin{array}{l}\text { Total } \\
{[n=432]}\end{array}$ & $\begin{array}{c}\text { EPSA } \\
\text { Urbano } \\
{[n=250]}\end{array}$ & $\begin{array}{l}\text { Rural } \\
{[n=182]}\end{array}$ & $\begin{array}{l}\text { Total } \\
{[n=432]}\end{array}$ & $\begin{array}{c}\text { ELCSA } \\
\text { Urbano } \\
{[\mathrm{n}=250]}\end{array}$ & $\begin{array}{l}\text { Rural } \\
{[n=182]}\end{array}$ \\
\hline & \multicolumn{6}{|c|}{ Hogares } \\
\hline Measure (DS) & - 3,95 (1,77) & $-4,31(1,66)$ & - 3,71 (1,97) & $-4,03(3,13)$ & $-5,39(3,63)$ & $-3,12(2,94)$ \\
\hline Infit (DS) & $0,98(0,41)$ & $0,93(0,51)$ & $0,99(0,38)$ & $0,91(0,72)$ & $0,77(1,04)$ & $0,97(0,54)$ \\
\hline Outfit (DS) & $0,91(1,11)$ & $0,91(1,09)$ & $0,87(1,08)$ & $0,89(1,70)$ & $0,90(1,88)$ & $0,82(1,54)$ \\
\hline Fiabilidad $*$ & 0,06 & 0,00 & 0,29 & 0,74 & 0,72 & 0,78 \\
\hline \multirow[t]{2}{*}{ Separación } & 0,24 & 0,00 & 0,64 & 1,67 & 1,62 & 1,90 \\
\hline & \multicolumn{6}{|c|}{ Ítems } \\
\hline Measure (DS) & $-0,71(2,87)$ & $-0,64(2,74)$ & $-0,66(2,80)$ & $0,00(2,53)$ & $0,00(2,78)$ & $0,00(2,49)$ \\
\hline Infit (DS) & $0,98(0,19)$ & $0,95(0,30)$ & $0,99(0,17)$ & $0,97(0,12)$ & $0,96(0,19)$ & $0,97(0,17)$ \\
\hline Outfit (DS) & $0,91(0,48)$ & $0,91(0,58)$ & $0,87(0,49)$ & $2,09(2,25)$ & $1,34(1,91)$ & $2,53(3,58)$ \\
\hline Fiabilidad & 0,95 & 0,93 & 0,94 & 0,99 & 0,98 & 0,98 \\
\hline Separación & 4,50 & 3,53 & 3,92 & 9,32 & 6,60 & 6,67 \\
\hline
\end{tabular}




\section{DISCUSIÓN}

Este estudio evaluó el desempeño de dos pruebas que han sido utilizadas en Colombia en estudios regionales y encuestas nacionales (19-21). Estudios previos han mostrado poca coherencia de los resultados sobre seguridad alimentaria a nivel del hogar frente a métodos referentes, y pobre reproducibilidad y validez $(3,11)$, éstos son contradictorios de otros que afirman las cualidades psicométricas de estas escalas y su utilidad en la política pública $(20,22)$. El sentido del análisis Rasch aquí realizado supera la exploración y alcanza el de la evaluación $(1,6,7)$. Además, se discute la implicación que sobre la política publica y el gasto nacional tiene el no medir adecuadamente el tema de la seguridad alimentaria en un país. Las pruebas fueron aplicadas a hogares de todos los estratos socioeconómicos, en diferentes zonas geográficas y con características medias similares a las reportadas en el último censo nacional de Colombia (23). Debido al diseño del estudio, la selección de los sujetos, el entrenamiento de los nutricionistas encuestadores y las técnicas y métodos de análisis estadísticos empleados se controlaron todas las posibles fuentes de sesgos de selección, información y confusión (1).

Sobre La EPSA. La inhabilidad de los hogares para contar con SA en relación con la dificultad de los ítems empleados para evaluarla evidenciada en la figura 1, la tabla 2, y los valores extremos de measure o dificultad de los ítems hacen que los resultados de la escala porten un alto grado de imprecisión. En la práctica la EPSA “mide" la inseguridad alimentaria con base en un solo ítem, el p1, el cual por su baja dificultad -8,46 logits, desviación estándar (DS) de 1,83-, resulta inapropiado para medir los hogares estudiados. Con base en los estadísticos calculados cinco de los doce ítems presentan sobreajuste y tres de los doce incluido el primero, degradación de la medición. Según el índice de fiabilidad la clasificación de la INSA en los hogares no es confiable (tabla 4), esto se debe a los pocos ítems con measure y estadísticos de ajuste adecuados. La alternativa para aumentar la confiabilidad de la clasificación del hogar no es viable dado que esta muestra cubrió todo el rango posible de habilidad de los hogares; todos los estratos socioeconómicos y las áreas urbana y rural. Con respecto a los ítems el nivel de dificultad es confiable, puede afirmarse que los hogares aportan más información a los ítems, que los ítems sobre los hogares, esto no es diferencial dado el área geográfica del hogar. Con base en los estadísticos calculados cinco de los doce ítems presentan sobreajuste y tres de los doce incluido el primero degradación en la medición, el $41,7 \%$ de los ítems necesitan revisión y tres de ellos $(25 \%)$ deberían eliminarse. El orden observado con base en la dificultad de los ítems no es el presentado en la EPSA comprometiendo la validez de constructo y el concepto subyacente de monotonía y dificultad progresiva de los ítems en la medición $(6,7)$. La EPSA es unidimensional según la figura 2 , confirmándose por el porcentaje de varianza inexplicada en el primer contraste. Dado que no se encontró análisis Rasch para la EPSA no es posible comparar estos hallazgos.

Sobre la ELCSA. Según la figura 1 la habilidad media de los hogares apenas alcanza la primera desviación estándar de la dificultad de los ítems propuestos, y la gran mayoría de ellos dada su habilidad para contar con SA se sitúan a dos desviaciones de la dificultad de los ítems. Con base en el oufit nueve ítems, entre ellos dos de los cuatro primeros, miden con alto grado de imprecisión. Al igual que con la EPSA p1 define el resultado en muy buena medida y la prueba resulta inapropiada para los hogares estudiados. Con base en los estadísticos calculados al menos cuatro de los quince ítems presentan sobreajuste y cuatro de los quince incluido el primero degradación de la medición. El 33,3\% de los ítems necesita revisión y cuatro de ellos $(26,7 \%)$ deberían eliminarse. Según el índice de fiabilidad la clasificación de la INSA en los hogares no alcanza a ser confiable $(<0,80)$, esto se debe como con la EPSA a los pocos ítems con measure y estadísticos de ajuste adecuados. Con respecto a los ítems el nivel de dificultad es confiable, los hogares aportan más información a los ítems, que los ítems sobre los hogares, esto no es diferencial dado el área geográfica del hogar. El orden observado con base en la dificultad de los ítems no es el presentado en la ELCSA, comprometiendo la validez de constructo y el concepto subyacente de monotonía y dificultad progresiva de los ítems en la medición (6, 7). La ELCSA es bidimensional. Los ítems p14 y p15 conforman una dimensión, confirmándose esto por el porcentaje de varianza inexplicada en el primer contraste, sin embargo p14 y p15 preguntan por aspectos que son complementarios. Este estudio encontró dos dimensiones mientras que los autores de la ELCSA revindican al menos cuatro; cantidad de alimentos, calidad y variedad de alimentos, incertidumbre frente a la disponibilidad de alimentos y hambre (22).

La ELCSA en una versión con 17 ítems y analizada por sus autores con el modelo Rasch mostró infit para todos los ítems en el rango de 0,8 a 1,2 y monotonía en la progresión de la dificultad de los ítems (22). Además, valores de measure predominantemente negativos y por encima de +-2.0. El análisis además se realizó para los ítems de adultos y de niños con los mismos resultados (22).

Dado los estadísticos presentados, las escalas eva- 
luadas no pueden considerarse como adecuadas para medir la seguridad alimentaria a nivel del hogar, incluso al evaluar el desajuste de los ítems de manera conservadora utilizando rangos de outfit entre 0,5 y 1,5 en vez de 0,8 a 1,2 . La validez de constructo esta comprometida al revaluarse la agrupación y el ordenamiento esperado de los ítems (tablas 2, 3 y figuras 1 y 2), en particular p1 en ambas escalas se caracteriza por su baja dificultad dada la habilidad de los hogares estudiados (figura 1).

En países como Colombia y en sus hogares promedio como los estudiados pertenecientes a la capital, una ciudad intermedia y zonas urbanas y rurales, medir sin precisión la inseguridad alimentaria compromete la cifra de prevalencia y la identificación de los hogares en donde debe focalizarse o hacia los cuales debe dirigirse la inversión y política pública. La medición utilizando herramientas que no son adecuadas pero si atractivas, puede ser un nuevo y mayúsculo problema que se sume a otras condiciones asociadas a la inseguridad alimentaria de los hogares; inequidad, concentración de la riqueza, transición demográfica, nutricional y epidemiológica y pobreza en aumento (12). Las dos pruebas evaluadas fueron usadas en 2005 y 2010 en encuestas nacionales en Colombia (ENSIN), por lo aquí expuesto es necesario revisar los resultados alcanzados y su uso de manera conservadora.

\section{RESUMEN}

Este estudio sometió dos escalas utilizadas para determinar la seguridad alimentaria en las encuestas nacionales de situación nutricional de Colombia (ENSIN) al modelo Rasch para conocer su desempeño ante la evidencia contradictoria de su utilidad. En la práctica la EPSA mide la inseguridad alimentaria con base en un solo ítem, el p1, el cual por su baja dificultad $-8,46$ logits, desviación estándar (DS) de 1,83-, resulta inapropiado para medir los hogares estudiados. Con base en los estadísticos calculados cinco de los doce ítems presentan sobreajuste y tres de los doce incluido el primero, degradación de la medición. Al igual que con la EPSA p1 define el resultado en muy buena medida y la prueba resulta inapropiada para los hogares estudiados. Con base en los estadísticos calculados al menos cuatro de los quince ítems presentan sobreajuste y cuatro de los quince incluido el primero degradación de la medición. La medición utilizando herramientas que no son adecuadas pero si atractivas, puede ser un nuevo y mayúsculo problema que se sume a otras condiciones asociadas a la inseguridad alimentaria de los hogares.

Palabras clave: Seguridad alimentaria; reproducibilidad de resultados; validez de las pruebas; hambre; Colombia.
Dirigir la correspondencia a:

Profesor

Oscar Fernando Herrán

Centro de Investigaciones Epidemiológicas

Facultad de Salud,

Universidad Industrial de Santander

Carrera 32 No. 29-31, tercer piso, oficina 304,

Bucaramanga, Colombia

Fax: (57-7) 6345781

E-mail: bscar herran@gmail.com

herran@uis.edu.co

Declaración de conflicto de interés: Los autores declaran que no tienen conflicto de interés de ningún tipo, ni real o potencial sobre los resultados presentados.

Agradecimientos: Este estudio fue posible por la cofinanciación del Departamento Administrativo de Ciencia, Tecnología e Innovación de Colombia (COLCIENCIAS), código; 110245921548 y de la Secretaria Distrital de Salud de Bogotá, la Gobernación de Cundinamarca, y las Universidades Nacional de Colombia e Industrial de Santander. Código interno UIS; 8677.

\section{BIBLIOGRAFÍA}

1. Orozco LC. Medición en salud; diagnóstico y evaluación de resultados. Bucaramanga, Colombia. Publicaciones UIS, 2010.

2. Chmura H. Evaluating medical test. London. SAGE Publications, 1992.

3. Herrán OF, Quintero DC, Prada GE. Seguridad alimentaria; un método alterno frente a uno clásico. Rev. Salud Pública. 2010;12:546-57.

4. Pelletier DL, Olson CM, Frongillo EA. Inseguridad alimentaria, hambre y desnutrición. En: Conocimientos actuales sobre nutrición. Octava edición. Washington, DC. OPS e Instituto Internacional de Ciencias de la Vida, 2003:762-75. Publicación científica y técnica No. 592.

5. Herrán OF. Quintero DC, Prada GE. Validez factorial, consistencia interna y reproducibilidad de la escala de seguridad alimentaria en hogares de Bucaramanga, Colombia. Rev Chil Nut. 2009;36:169-79.

6. Bond TG, Fox CM. Applying the Rasch Model; fundamental measurement in the human sciences. New Jersey. Second edition. Lawrence Erlbaum Associates, Publishers, 2007

7. Alagumalai S, Curtis DD, Hungi N. (Editors). Applied Rasch measurement: A Book of exemplars. Netherlands. Springer, 2005.

8. Instituto Colombiano de Bienestar Familiar (ICBF). Encuesta Nacional de la Situación Nutricional en 
Colombia, 2005. Bogotá, Colombia, 2005.

9. Instituto Colombiano de Bienestar Familiar (ICBF). Encuesta Nacional de la Situación Nutricional en Colombia, 2010. Bogotá, Colombia, 2010.

10. Herrán OF, Quintero DC, Prada GE. Seguridad alimentaria; un método alterno frente a uno clásico. Rev. Salud Pública. 2010;12:546-57.

11. Del Castillo SE, Jiménez AZ, Camargo M, Herrán OF. Calidad de la dieta, percepción del hambre y seguridad alimentaria en población urbana y rural: estudio multicéntrico de evaluación de tecnología diagnóstica en Cundinamarca, Bogotá, Bucaramanga y su área metropolitana. Bucaramanga, Colombia: Informe a COLCIENCIAS, proyecto código: 110245921548. Universidad Industrial de Santander, 2011. (Informe Técnico)

12. Del Castillo SE (Editora). Observatorio de seguridad alimentaria y nutricional (OBSAN): cinco años de trayectoria, reflexiones 2005-2010. Bogotá. Universidad Nacional de Colombia, 2010:23-50.

13. Leisinger KM, Schmitt KM, Pandya-Lorch R. Six billion and counting: Population and food security in the 21 st century. Washington, D.C. Internat Food Policy Res Institute 2002:57-76.

14. Hair JF, Anderson RE, Tatham RL, Black WC. Análisis multivariante. Quinta edición, Madrid: Prentice Hall, 1999.

15. Kline P. An easy guide to factor analysis. London: Routledge Press, 1994;157-84.

16. STATA. Estimation of means, totals, ratios, and proportions for survey data. Stata Technical Bul- letins. 1996;6:213-35.

17. StataCorp, 2008. Stata Statistical Software: Release 10.1. Collegue Station, TX: StataCorp LP.

18. Linacre JM, 2011. Winsteps Rasch Measurement. Release 3.72.3.

19. Herrán OF. Quintero DC, Prada GE. Validez factorial, consistencia interna y reproducibilidad de la escala de seguridad alimentaria en hogares de Bucaramanga, Colombia. Rev Chil Nutr 2009;36:169-79.

20. Álvarez MC, Estrada A, Montoya EC, MelgarQuiñonez H. Validación de escala de percepción de la seguridad alimentaria doméstica en Antioquia, Colombia. Salud Pública Mex. 2006;48:474-81.

21. Alvarado BE, Zunzunegui MV, Delisle H. Validación de escalas de seguridad alimentaria y de apoyo social en una población afro-colombiana: aplicación en el estudio de prevalencia del estado nutricional en niños de 6 a 18 meses. Cad Saúde Pública. 2005;21:724-36.

22. Instituto Colombiano de Bienestar Familiar (ICBF), Organización de las Naciones Unidas para la Agricultura y la Alimentación (FAO), Departamento Administrativo Nacional de Estadística (DANE), Universidad de Antioquia Adaptación y validación interna y externa de la escala Latinoamericana y Caribeña para la medición de seguridad alimentaria en el hogar - ELCSA Colombia-, 2008. (Informe Técnico)

23. Departamento Nacional de Estadística (DANE) Censo 2005 Conombia. Disponible en: http://www dane.gov.co/censo/. Consultado en Agosto de 2011.

\footnotetext{
APÉNDICE 1

Ítems de las escalas evaluadas.

\section{EPSA.}

p1. En el último mes falto dinero para comprar alimentos?

p2. En el ultimo mes en el hogar se disminuyo el numero de comidas como dejar de desayunar, almorzar o comer por falta de dinero para comprar alimentos?

p3. En el ultimo mes algún adulto del hogar comió menos de lo que deseaba por falta de dinero para comprar alimentos?

p4. En el ultimo mes algún adulto dejo de desayunar, de almorzar o de comer por falta de dinero para comprar alimentos?

p5. En el último mes algún adulto comió menos en la comida principal porque la comida no alcanzó para todos?

p6. En el último mes algún adulto se quejó de hambre por falta de alimentos en el hogar?

p7. En el último mes algún adulto se acostó con hambre porque no alcanzó el dinero para la comida?

p8. En el último mes se compraron menos indispensables para los jóvenes y los niños porque el dinero no alcanzó?

p9. En el último mes algún joven o niño dejó de desayunar, almorzar o comer por falta de dinero para comprar alimentos?

p10. En el último mes algún joven o niño dejó comió menos en la comida principal porque la comida no alcanzó para todos?

p11. En el último mes algún joven o niño se quejó de hambre por falta de alimentos en el hogar?

p12. En el último mes algún joven o niño se acostó con hambre porque no alcanzó el dinero para la comida?
} 


\section{Continuación APÉNDICE 1}

\section{ELCSA.}

p1. En los últimos 30 días, ¿Usted se preocupo alguna vez de que en su hogar se acabaran los alimentos debido a la falta de dinero?

p2. En los últimos 30 días, ¿Alguna vez en su hogar se quedaron sin alimentos por falta de dinero?

p3. En los últimos 30 días, ¿Alguna vez en su hogar se quedaron sin dinero para obtener una alimentación nutritiva; es decir que contenga carne leche ó productos lácteos, frutas huevos, verduras, cereales, leguminosas, tubérculos y plátanos?

p4. En los últimos 30 días, ¿Alguna vez usted o algún adulto de su hogar dejó de desayunar, almorzar o comer por falta de dinero?

p5. En los últimos 30 días, ¿Alguna vez usted o algún adulto de su hogar no pudo variar la alimentación por falta de dinero?

p6. En los últimos 30 días, ¿Alguna vez usted o algún adulto de su hogar comió menos de lo que esta acostumbrado por falta de dinero?

p7. En los últimos 30 días, ¿Alguna vez usted o algún adulto de su hogar sintió o se quejó de hambre y no comió por falta de dinero?

p8. En los últimos 30 días, ¿Alguna vez usted o algún adulto de su hogar solo comió una vez al día o dejó de comer en todo un día por falta de dinero?

p9. En los últimos 30 días, ¿Alguna vez algún adulto de su hogar se acostó con hambre porque no alcanzó el dinero para los alimentos?

p10. En los últimos 30 días, ¿Alguna vez por falta de dinero algún niño o joven de su hogar dejó de tener una alimentación nutritiva; es decir, que contenga carne leche ó productos lácteos, frutas, huevos, verduras, cereales, leguminosas, tubérculos y plátanos?

p11. En los últimos 30 días, ¿Alguna vez por falta de dinero algún niño o joven de su hogar no pudo variar la alimentación por falta de dinero?

p12. En los últimos 30 días, ¿Alguna vez usted tuvo que disminuir la cantidad servida en las comidas de algún niño o joven de su hogar por falta de dinero?

p13. En los últimos 30 días, ¿Alguna vez algún niño o joven de su hogar se quejó de hambre pero no se pudo comprar más alimentos por falta de dinero?

p14. En los últimos 30 días, ¿Alguna vez algún niño o joven de su hogar se acostó con hambre porque no alcanzó el dinero para los alimentos?

p15. En los últimos 30 días, ¿Alguna vez algún niño o joven de su hogar solo comió una vez al día o dejó de comer todo un día por falta de dinero? 